\title{
Aptidão física e composição corporal de alunos de ensino médio militar: um estudo transversal
}

Physical fitness and body composition of military high school students: a cross-sectional study

\author{
Priscila dos Santos Bunn ${ }^{1,2, *}$ \\ Bruno de Souza Terra ${ }^{1}$ \\ Allan Inoue Rodrigues ${ }^{1}$ \\ Diego Correia da Paschoa ${ }^{3}$ \\ Maria Elisa Koppke Miranda ${ }^{1}$ \\ Carlos Henrique Moreira ${ }^{3}$ \\ Mariana dos Santos Menezes ${ }^{3}$ \\ Daniel Alves de Souza ${ }^{1}$
}

\begin{abstract}
Resumo
Objetivo: avaliar se a divisão em grupos pelo desempenho no Teste de Cooper (TC) estratifica a composição corporal e o desempenho em testes de força, em alunos de ensino médio. Métodos: 112 alunos foram submetidos a avaliações antropométricas no início do curso de ensino médio militar. Realizaram os testes de salto horizontal bilateral (TSHB), salto unilateral, flexões na barra, abdominais com apoio, flexões de braço e TC. A associação entre as variáveis físicas e antropométricas e o TC foi avaliada pela análise de Correlação de Pearson e a análise de regressão linear múltipla. Para comparar os tercis (T1, T2 e T3) nos diferentes indicadores físicos, foi utilizado a ANOVA de uma entrada. Resultados: Verificou-se uma correlação fraca a moderada entre o TC e as variáveis IMC, perimetria de cintura e percentual de gordura e no desempenho nos testes de salto horizontal, barras e flexões, com $R=0,511$ e $R^{2}=0,26$ (percentual de gordura e desempenho nos testes de salto bilateral, barra, flexão e abdominal como variáveis independentes). Houve diferença significativa entre o grupo T3 e T1 nos testes de barra, flexão, no percentual de gordura e perimetria abdominal, e para IMC e TSHB, houve diferença entre T3 e T1 e T3 e T2. Conclusão: A divisão dos alunos em grupos pelo desempenho no TC estratifica também indivíduos com menor IMC e maiores distâncias no TSHB, embora não pareça ser suficiente para predizer o desempenho no TC.
\end{abstract}

Palavras-chave: militares, condicionamento físico, aptidão física.

\section{Abstract}

Objective: to evaluate whether the division into groups by performance in the Cooper Test (CT) stratifies body composition and performance in force tests in high school students. Methods: 112 students were submitted to anthropometric assessments at the beginning of the military high school course. They performed the standing long jump test (SLJT), unilateral jump, pull-up, curl-up, push-up and CT. The association between physical and anthropometric variables and CT was evaluated by Pearson's correlation analysis and multiple linear regression analysis. To compare the tertiles (T1, T2 and T3) in the different physical indicators, the ANOVA of one entry was used. Results: There was a weak to moderate correlation between $\mathrm{CT}$ and BMI variables, waist perimetry and fat percentage, and performance in SLJT, pull-up and push-up tests, with $R=0.511$ and $R 2=0.26$ (percentage of fat and performance in the tests of SLJT, pull-up, curl-up, push-up as independent variables). There was a significant difference between the T3 and T1 groups in the pull-up tests, flexion, percentage of fat and waist perimetry, and for BMI and BHT, there were differences between T3 and T1 and T3 and T2. Conclusion: The division of students into groups by CT performance also stratifies individuals with lower $\mathrm{BMI}$ and greater distances in SLJT, although this does not appear to be enough to predict CT performance.

Keywords: military personnel, physical conditioning, physical fitness.
Afiliação dos autores

${ }^{1}$ Centro de Educação Física Almirante Adalberto Nunes, Marinha do Brasil;

${ }^{2}$ Programa de Pós-Graduação em Ciências do Exercício e do Esporte, Universidade do Estado do Rio de Janeiro; Rio de Janeiro, Brasil.

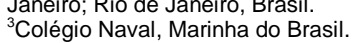

${ }^{*}$ Autor correspondente

Vila Edson Passos, 40, Mesquita, CEP: 26585-200, Rio de Janeiro, Brasil,

e-mail:priscilabunn@yahoo.com.br

Conflito de interesses

Os autores declararam não haver conflito de interesses.

Processo de arbitragem

Recebido: 13/12/2017 Aprovado: 30/01/2018 


\section{Introdução}

O consumo máximo de oxigênio (VO2máx) é a mais alta taxa de oxigênio consumida para realizar um esforço máximo e permite a avaliação da aptidão física dos indivíduos, orienta a prescrição do exercício e tem relação com o risco cardiovascular 1,2. O "padrão-ouro" para a avaliação direta do VO2máx é o teste de ergoespirometria, em que são avaliadas as frações expiradas dos gases respiratórios durante esforço máximo $3^{4}$.

Como alternativa a situações que impossibilitem o uso da ergoespirometria, foram desenvolvidos métodos de avaliação indireta do VO2máx, como o Teste de Cooper (TC), baseado em uma equação de predição com múltiplas variáveis de entrada 3,4 . O TC é um teste de corrida amplamente utilizado, pois possui boa correlação com o VO2máx medido de forma direta e tem baixo custo e fácil exequibilidade ${ }^{4}$. Este teste comumente é aplicado em atletas, militares, e outros grupos, quando o objetivo é avaliar o condicionamento aeróbio, em especial em avaliações de grandes grupos ${ }^{4,5}$. Outro protocolo de testes amplamente utilizado em cursos militares é o Fitness "Tests" " Em adolescentes, a aptidão física é influenciada por fatores como a força/resistência abdominal, o condicionamento aeróbio e a composição corporal ${ }^{8}$. Outros autores consideram que a composição corporal é fortemente correlacionada com o condicionamento aeróbio ${ }^{9-11}$. Entretanto, é comum a recomendação da divisão das turmas de alunos exclusivamente pelo desempenho no TC, em especial no militarismo.

Os valores de referência para o TC, bem como sua relação com a composição corporal e a força muscular ${ }^{12-14}$, são bem estabelecidos para alunos de cursos de formação militar adultos europeus ${ }^{5,6}$. Entretanto, anualmente, centenas de adolescentes brasileiros iniciam a carreira em colégios militares de nível médio, e a relação entre o TC, Fitness Tests e composição corporal é desconhecida para esta população. Desta forma, o objetivo do presente estudo foi avaliar se a divisão em grupos pelo desempenho neste teste estratifica também a composição corporal e o desempenho em outros testes físicos, em alunos de ensino médio militar, bem como analisar quais são as variáveis físicas e de composição corporal que predizem o desempenho no TC.

\section{Métodos}

\section{Participantes}

Foram convidados para participar do estudo todos os alunos do primeiro ano do Colégio Naval, a escola de ensino de nível médio da Marinha do Brasil. A amostra consistiu de indivíduos do sexo masculino, com idade entre 15 e 18 anos ( $16 \pm 1$ anos, $67,5 \pm 11,0 \mathrm{~kg}$ e $173,2 \pm 5,5 \mathrm{~cm}$ ), que estivessem nas duas primeiras semanas do curso (fase de adaptação à rotina militar) e que não apresentassem lesões ou procedimentos cirúrgicos recentes que impossibilitassem a realização dos testes. Este estudo foi aprovado pelo Comitê de Ética em Pesquisa do Hospital Naval Marcílio Dias (CAAE 50405615.4.0000.5256).

\section{Desenho do estudo}

Este é um estudo transversal, redigido segundo as recomendações do StrobeStatement ${ }^{15}$, que faz parte de um estudo de coorte de predição de lesões. Os responsáveis legais dos alunos receberam o convite para participar do estudo antes do início do curso. Após a assinatura dos termos de consentimento e de assentimento, os alunos foram submetidos a uma breve anamnese a respeito de doenças e lesões musculoesqueléticas que impossibilitassem a realização dos testes. Na primeira visita, após a verificação dos critérios de elegibilidade, foram medidas a estatura, a massa corporal, e calculado o Índice de Massa Corporal (IMC). O IMC foi classificado de acordo com o seguinte critério ${ }^{16}$ : baixo peso $(<$ $\left.18,5 \mathrm{~kg} \cdot \mathrm{m}^{-2}\right)$, normal $\left(18,5\right.$ a $\left.24,9 \mathrm{~kg} \cdot \mathrm{m}^{-2}\right)$, sobrepeso (25 a 29,9 $\left.\mathrm{kg} \cdot \mathrm{m}^{-2}\right)$, ou obesidade $\left(\geq 30 \mathrm{~kg} \cdot \mathrm{m}^{-2}\right)$. O percentual de gordura foi estimado aplicando as equações de Jackson e Pollock de três dobras cutâneas, para maiores de 18 anos ${ }^{17}$ e Slaugther de duas dobras cutâneas, para menores de 18 anos ${ }^{18}$. A perimetria de cintura foi realizada na altura do menor perímetro entre 0 apêndice xifoide e a cicatriz umbilical, e o resultado graduado de acordo com o seguinte critério ${ }^{16}$ : magro $(<80 \mathrm{~cm})$, mediano (80 a $93,5 \mathrm{~cm})$, aumentada (94 a $101,5 \mathrm{~cm}$ ) ou alta $(\geq$ $102 \mathrm{~cm})$.

Posteriormente, foram realizados os testes de força muscular: salto horizontal bilateral, salto horizontal unilateral, flexão de braços, abdominal e barra ${ }^{6}$. O salto horizontal bilateral foi realizado com o participante de pé, com as pernas paralelas e afastadas aproximadamente a distância entre os ombros. $\mathrm{O}$ movimento de membros superiores era livre, com o intuito de favorecer o impulso para o salto, e o indivíduo saltar a maior distância possível em três tentativas. Para o salto horizontal unilateral, o movimento foi iniciado com o participante de pé e com apoio unipodal. O movimento de membros superiores também foi livre, com o mesmo intuito do teste anterior. $O$ indivíduo saltou a maior distância possível com apoio unilateral e aterrisagem bilateral, em três tentativas em cada lado e calculou-se a diferença percentual entre as médias das distâncias dos lados direito e esquerdo. Nos demais testes, os indivíduos realizaram o máximo de repetições em 60 segundos. Para realizar a flexão de braços, o participante iniciou o movimento em decúbito ventral, as mãos posicionadas no solo na direção dos ombros e com os cotovelos flexionados. Posteriormente, realizou-se a extensão completa de cotovelos mantendo o corpo ereto, e retornou à posição inicial. O teste de abdominal foi iniciado com o participante em decúbito dorsal com as mãos entrelaçadas na região posterior do pescoço e os joelhos em flexão de $90^{\circ}$. Um avaliador estabilizou os tornozelos. O indivíduo realizou a flexão de tronco até os cotovelos tocarem os joelhos e, posteriormente, retornar à posição inicial em decúbito dorsal. Durante o teste de barra, o participante foi solicitado a elevar o seu queixo acima de uma barra fixa e retornar à posição inicial com os cotovelos completamente estendidos, sem apoio no solo ${ }^{6}$. Por fim, após 24 horas, foi realizada a segunda visita, que consistiu no TC, em que o participante deveria correr a maior distância possível em uma pista de atletismo de $244 \mathrm{~m}$, durante 12 minutos.

\section{Análise Estatística}

Para a análise multivariada, foi utilizada a regressão linear múltipla, sendo a distância em metros no TC considerada como variável dependente. A metodologia empregada na análise multivariada foi a inclusão inicial de todas as variáveis significativas na análise univariada pela análise da Correlação de Pearson e a posterior exclusão, uma a uma, daquelas que não apresentavam contribuição significativa para o modelo (método backward) ou que apresentassem multicolinearidade ${ }^{19}$. Utilizou-se como variáveis independentes o IMC, percentual de gordura, perímetro de cintura, flexão de braços, barra, abdominais, salto bilateral e a diferença entre os lados no salto unilateral.

$O$ TC teve o seu resultado organizado em ordem decrescente de desempenho e foi classificado em três grupos (tercis): T1, T2 e T3. Para comparar os grupos nos diferentes indicadores físicos, foi utilizado a ANOVA de uma entrada, com post hoc de Bonferroni.

$\mathrm{Na}$ análise dos dados utilizou-se o programa SPSS (versão 23.0, International Business Machines (IBM), Estados Unidos.), considerando-se o nível de significância de 5\%.

\section{Resultados}

Foram avaliados 121 alunos para a elegibilidade. O diagrama de fluxo de participantes incluídos está na Figura 1

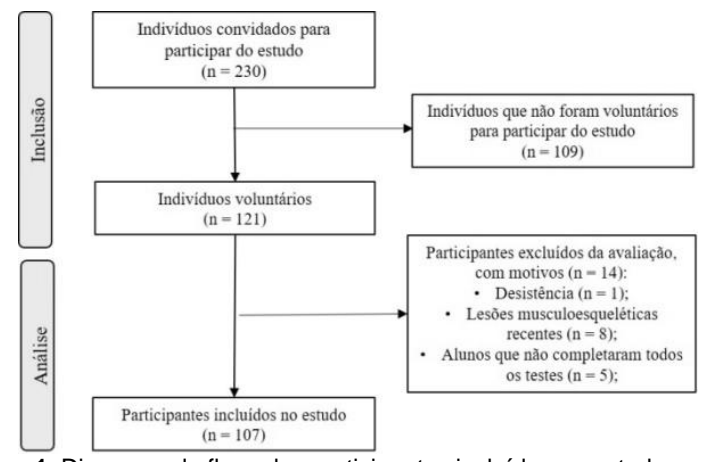

Figura 1. Diagrama de fluxo dos participantes incluídos no estudo. 
A Tabela 1 apresenta os resultados da Correlação de Pearson entre o TC e cada uma das variáveis preditoras ou independentes. Inicialmente foram inseridas na análise de regressão todas as variáveis com correlação significativa com o TC.

\section{Tabela 1}

Resultados da Correlação de Pearson entre testes físicos e antropométricos e o Teste de Cooper.

\begin{tabular}{lcc}
\hline \multicolumn{1}{c}{ Variáveis preditoras } & $\mathrm{r}$ & $P$-valor \\
\hline IMC & 0,424 & $<0,001$ \\
\% Gordura corporal & 0,465 & $<0,001$ \\
Perimetria de cintura & 0,303 & 0,001 \\
Salto horizontal bilateral & 0,330 & 0,001 \\
\% Salto horizontal unilateral & 0,024 & 0,807 \\
Barras em 1 min & 0,387 & $<0,001$ \\
Abdominais em 1 min & 0,243 & 0,012 \\
Flexões de braço em 1 min & 0,305 & 0,001 \\
\hline IMC = índice de massa corporal; $r$ = coeficiente de correlação de Pearson; \%
\end{tabular}

Salto horizontal unilateral = diferenca percentual entre as distâncias médias cada membro no teste de salto horizontal unilateral.

Na Tabela 2 estão relacionados os resultados da análise do modelo de regressão da associação entre o TC e testes físicos e de composição corporal que apresentou o melhor Coeficiente de Determinação $\left(R^{2}\right)$. Após a análise da multicolinearidade, foi realizada a exclusão da variável IMC da análise. O detalhamento das variáveis da equação do modelo selecionado está na Tabela 3. A Tabela 4 apresenta os resultados dos grupos (estratificados por tercis, de acordo com o desempenho no TC) de perimetria de cintura, IMC, composição corporal, testes de força e TC.

Tabela 2

Resultados da regressão da associação entre o Teste de Cooper e testes físicos e de composição corporal.

\begin{tabular}{lllll}
\hline \multicolumn{1}{c}{ Variáveis dependentes } & $R$ & $R^{2}$ & $\begin{array}{c}\text { EPE } \\
(\mathrm{m})\end{array}$ & $P$-valor \\
\hline $\begin{array}{l}\text { \% gordura corporal, perimetria de } \\
\text { cintura, flexões de braço em 1 min, }\end{array}$ & 0,511 & 0,26 & 295,92 & $<0,001$ \\
abdominais, salto horizontal \\
bilateral, barras em 1 min *
\end{tabular}

Tabela 3

Modelo de regressão da associação entre o TC e testes físicos e de composição corporal.

\begin{tabular}{lcccc}
\hline \multicolumn{1}{c}{ Teste de Cooper } & Beta & EPE & $T$ & $P$-valor \\
\hline Constante & 2090,17 & 470,85 & 4,44 & $<0,001$ \\
\% Gordura corporal & $-1,16$ & 6,34 & $-2,38$ & 0,02 \\
Perimetria de cintura & $-1,51$ & 5,39 & $-0,22$ & 0,83 \\
Flexões de braço em 1 min & 0,47 & 4,55 & 0,10 & 0,91 \\
Abdominais em 1 min & 4,44 & 4,04 & 1,09 & 0,27 \\
Salto horizontal bilateral & 2,55 & 2,39 & 1,06 & 0,29 \\
Barras em 1 min & 9,84 & 10,31 & 0,95 & 0,34 \\
\hline \%EPE = erro padrão da estimativa. & & &
\end{tabular}

Tabela 4

Resultados dos grupos separados por tercis de acordo com o desempenho no Teste de Cooper

\begin{tabular}{|c|c|c|c|c|}
\hline \multirow[b]{2}{*}{ Variáveis } & \multicolumn{3}{|c|}{ Grupos/Tercis } & \multirow[b]{2}{*}{$\begin{array}{r}P \text {-valor } \\
\text { ANOVA }\end{array}$} \\
\hline & $\begin{array}{c}\mathrm{T} 1 \\
(\mathrm{n}=37)\end{array}$ & $\begin{array}{c}\text { T2 } \\
(n=38)\end{array}$ & $\begin{array}{c}\text { T3 } \\
(n=37)\end{array}$ & \\
\hline Distância Cooper (m) & $2583,7 \pm 124,3$ & $2299,0 \pm 65,3^{*}$ & $1871,9 \pm 229,9^{* \star}$ & $<0,001$ \\
\hline IMC $\left(\mathrm{kg} \cdot \mathrm{m}^{-2}\right)$ & $21,2 \pm 2,3$ & $22,2 \pm 3,1$ & $24,0 \pm 3,5^{*, \star \star}$ & $<0,001$ \\
\hline$\%$ Gordura corporal (\%) & $12,9 \pm 4,6$ & $16,1 \pm 6,4$ & $19,2 \pm 7,2^{*}$ & $<0,001$ \\
\hline Perímetro de cintura $(\mathrm{cm})$ & $72,1 \pm 5,4$ & $74,6 \pm 7,2$ & $77,0 \pm 7,6^{*}$ & 0,010 \\
\hline Salto horizontal $(\mathrm{cm})$ & $195,1 \pm 18$ & $189,9 \pm 26,8$ & $176,5 \pm 24,5^{\star * * *}$ & 0,003 \\
\hline Barras em 1 min (rep) & $7 \pm 5$ & $5 \pm 4$ & $3 \pm 3^{*}$ & $<0,001$ \\
\hline Abdominais em $1 \mathrm{~min}$ (rep) & $37 \pm 7$ & $35 \pm 7$ & $33 \pm 9$ & 0,076 \\
\hline Flexões de braço em 1 min (rep) & $30 \pm 9$ & $26 \pm 8$ & $23 \pm 8^{*}$ & 0,006 \\
\hline
\end{tabular}

Post hoc de Bonferroni indicando: ${ }^{*}=$ diferente de $\mathrm{T} 1 ;{ }^{*}=$ diferente de T2;

$\mathrm{IMC}=$ índice de massa corporal; $\% \mathrm{G}=$ percentual de gordura .

\section{Discussão}

Os resultados sugerem que os alunos com melhor desempenho no TC apresentam melhores variáveis antropométricas (IMC, percentual de gordura e perímetro de cintura), bem como melhor desempenho nos testes de salto horizontal bilateral, abdominais, barra e flexão. Entretanto, a correlação destas variáveis com o TC variou de fraca a moderada (Tabela 1) ${ }^{20}$ e não houve associação da diferença percentual entre as distâncias médias de cada membro nos saltos horizontais unilaterais e o TC.

Considerando-se todas as variáveis físicas e antropométricas que possuem correlação com o TC como variáveis independentes na análise multivariada, verifica-se que apenas $26 \%$ do desempenho no TC é explicado pelo melhor modelo de regressão desenvolvido (Tabela 2). Possivelmente, existem outras variáveis não avaliadas no presente estudo que correspondem a aproximadamente $74 \%$ do desempenho. Um fator contribuinte para os resultados encontrados, pode ter sido o tamanho amostral, com consequente impacto na robustez da análise ${ }^{21}$. No presente estudo, apenas o percentual de gordura contribuiu significativamente para o modelo de regressão (Tabela 3). Uma possível causa pode ser a sua melhor correlação com o TC $(r=0,46)$ dentre todas as variáveis independentes estudadas.

Avaliando-se os alunos em tercis (Tabela 4), de acordo com o desempenho no TC, verifica-se que os alunos com melhores percentuais de gordura, IMC, perimetria abdominal, barras e flexões (T3) apresentam melhores resultados no TC em relação ao grupo $\mathrm{T} 1$, não sendo diferente de T2. Os alunos com menores valores de IMC e maiores distâncias no salto horizontal bilateral (T3) percorreram maiores distâncias no TC, com diferenças significativas em relação aos outros dois grupos (T1 e T2). Não houve diferença significativa com o resultado no teste abdominal.

As valências físicas força e potência muscular podem influenciar no desempenho cardiorrespiratório. Jovens que praticam treinamento de resistência conseguem suportar maiores níveis de treinamento e possuem um menor risco de sofrer lesões musculoesqueléticas 22,23. Possivelmente, os indivíduos melhor condicionados possuem maior resistência à fadiga muscular durante as atividades do curso ${ }^{24-26}$, em que são realizados diversos tipos de exercícios físicos, como corrida, marcha e ordem unida, cujas valências principais são a resistência muscular e o condicionamento aeróbico 5,7,27 Portanto, separando-se os alunos pertencentes ao T3 do restante do grupo (T1 e T2) pode permitir estratégias de treinamento específicas para cada grupo.

Em jovens, a avaliação das espessuras das dobras cutâneas e IMC é considerada uma boa estimativa da composição corporal e são importantes índices relacionados à saúde. Já a perimetria abdominal é uma medida válida para estimar gordura corporal central ${ }^{28}$. Ao mesmo tempo, indivíduos que praticam atividades esportivas regulares e com melhor desempenho possuem um maior percentual de massa magra e um menor percentual de gordura ${ }^{10,11}$. Entretanto, não foram encontrados estudos que avaliaram a associação do TC com a composição corporal e o desempenho em outros testes físicos em alunos de colégios militares brasileiros, compostos por adolescentes. Em bombeiros militares adultos, verificou-se uma alta associação da composição corporal com o desempenho no TC, porém não foram realizados testes físicos ${ }^{9,29}$. Entretanto, em decorrência das diferentes faixas etárias avaliadas e da maturação dos participantes, tais resultados não podem ser comparados.

\section{Pontos fortes e limitações do estudo}

O presente estudo foi o primeiro a avaliar a associação entre o TC e os testes físicos em adolescentes de curso de formação militar, subsidiando a divisão dos alunos em três grupos para realizar o treinamento de força para membros inferiores e de dois grupos, para membros superiores. Entretanto, algumas limitações podem ser destacadas. Primeiro, não foi possível 
avaliar todos os 230 alunos do curso, em decorrência do não recebimento do Termo de Consentimento Livre e Esclarecido assinado pelos responsáveis legais. O ideal seria avaliar uma amostra mais representativa e com um maior tamanho amostral, tal como realizado em estudos europeus ${ }^{5,6}$. Além disso, não foi realizada a avaliação da maturação dos alunos, o que pode ser um viés de confundimento, visto que impacta na realização de tarefas motoras na adolescência ${ }^{30}$.

\section{Conclusão}

Apesar das limitações, os resultados deste estudo podem subsidiar a importância do treinamento de força, já que estes estão associados com 0 desempenho no TC. Os grupos pertencentes ao T3 e T2 poderiam ser um único grupo para o treinamento de força para membros superiores, separados de T1. Já para os membros inferiores, sugere-se que T3 realize o treinamento separado de T1 e T2. Os alunos com melhor desempenho no TC apresentam melhores variáveis antropométricas e melhor desempenho nos testes de salto horizontal bilateral, abdominais, barra e flexão. Entretanto, apenas este conjunto de variáveis parece não ser adequado para predizer o desempenho no TC.

\section{Referências}

1. Rodrigues AN, Perez AJ, Carletti L, Bissoli NS, Abreu GR. Maximum oxygen uptake in adolescents as measured by cardiopulmonary exercise testing: a classification proposal. J Pediatr (Rio J) [Internet]. testing: a classification proposal. J Pediatr (Rio J) [Internet].
2006;82(6):426-30. 2006;82(6):426-30. Available
http://www.jped.com.br/conteudo/Ing_resumo.asp?varArtigo=1533\&cod=\&i dSecao $=4$

1- Cordeiro V, Filho B, Lopes S, Bozza R, Rech CR, Campos W De. Correlates of Cardiorespiratory and Muscular fitness among Brazilian adolescents. :42-52.

2- Kravchychyn A, Alves J, Kravchychyn T, Nogueira G, Machado F. Comparação Entre Os Métodos Direto E Indireto De Determinação Do Vo 2Máx. Rev Bras Med do Esporte. 2011;17(4):270-3.

3- Bandyopadhyay A. Validity of Cooper's 12-minute run test for estimation of maximum oxygen uptake in male university students. Biol Sport. 2015;32(1):59-63.

4- Taanila H, Suni JH, Kannus P, Pihlajamäki H, Ruohola J-P, Viskari J, et al. Risk factors of acute and overuse musculoskeletal injuries among young conscripts: a population-based cohort study. BMC Musculoskelet Disord $\begin{array}{llll}\text { [Internet]. } & 2015 ; 16(1): 104 . \quad \text { Available } & \end{array}$ http://www.biomedcentral.com/1471-2474/16/104

5- Santtila M, Kyrölainen H, Vasankari T, Tiainen S, Palvalin K, H??Kkinen A, et al. Physical Fitness Profiles in Young Finnish Men during the Years 1975-2004. Med Sci Sport Exerc [Internet]. 2006;38(11):1990-4. Available from:

http://content.wkhealth.com/linkback/openurl?sid=WKPTLP:landingpage\&a $\mathrm{n}=00005768-200611000-00016$

6- Taanila $H$, Suni J, Pihlajamäki $H$, Mattila VM, Ohrankämmen $O$, Vuorinen $P$, et al. Musculoskeletal disorders in physically active conscripts: a oneyear follow-up study in the Finnish Defence Forces. BMC Musculoskelet

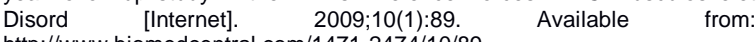
http://www.biomedcentral.com/1471-2474/10/89

7- Andreasi V, Michelin E, Rinaldi AEM, Burini RC. Physical fitness and associations with anthropometric measurements in 7 to 15 -year-old school children. J Pediatr (Rio J) [Internet]. 2010;0(0):497-502. Available from: $\mathrm{http} / / / \mathrm{jped} . \mathrm{com} . \mathrm{br} /$ conteudo/Ing_resumo.asp?varArtigo=2136\&cod=\&idSec $\mathrm{ao}=1$

8- Nogueira EC, Porto LGG, Nogueira RM, Martins WR, Fonseca RMC, Lunardi CC, et al. Body Composition is Strongly Associated With Cardiorespiratory Fitness in a Large Brazilian Military Firefighter Cohort. J Strength Cond Res [Internet]. 2016;30(1):33-8. Available from: http://content.wkhealth.com/linkback/openurl?sid=WKPTLP:landingpage\&a $\mathrm{n}=00124278-201601000-00005$

9- Neto AS, Paulo L, Mascarenhas G, Bozza R, Ulbrich AZ, Vasconcelos IQA, et al. VO2Max e composição corporal durante a puberdade: comparação entre praticantes e não praticantes de treinamento sistematizado de futebol. Rev Bras Cineantropometria Desempenho Hum. 2007;9(2):15964.

10- Mascarenhas LPG, Stabelini Neto A, Bozza R, Cezar CJ, Campos W de Comportamento do consumo máximo de oxigênio e da composição corporal durante o processo maturacional em adolescentes do sexo masculino participantes de treinamento de futebol. Rev Bras Ciência e Mov. 2006;14(2):49-56

11- Mayer JM, Nuzzo JL, Chen R, Quillen WS, Verna JL, Miro R, et al. The impact of obesity on back and core muscular endurance in firefighters. $J$ Obes. 2012;2012.

12- Gribble PA, Terada M, Beard MQ, Kosik KB, Lepley AS, McCann RS, et al Prediction of Lateral Ankle Sprains in Football Players Based on Clinical Tests and Body Mass Index. Am J Sports Med [Internet]. 2015;460-7. Available from: http://www.ncbi.nlm.nih.gov/pubmed/26646517

13- Teyhen DS, Shaffer SW, Butler RJ, Goffar SL, Kiesel KB, Rhon DI, et al. What Risk Factors Are Associated With Musculoskeletal Injury in US Army Rangers? A Prospective Prognostic Study. Clin Orthop Relat Res. 2015;473(9):2948-58.

14- Elm E Von, Altman DG, Egger M, Pocock SJ, Gøtzsche PC. The Strengthening the Reporting of Observational Studies in Epidemiology STROBE ) Statement: Guidelines for Reporting Observational Studies. PLoS One. 2007;4(10):1623-7.

15- World Health Organization (WHO). Obesity: preventing and managing the global epidemic. Report of a WHO consultation. World Health Organ Tech Rep Ser [Internet]. 2000;894:i-xii, 1-253. Available from: http://www.ncbi.nlm.nih.gov/pubmed/11234459

16- Jackson AS, Pollock ML. Generalized equations for predicting body density of men. Br J Nutr. 1978;40(3):497-504.

17- Slaughter, M.H.; Lohman, T.G.; Boileau, R.A.; Horswill, C.A.; Stillman, R.J.; Van Loan MD. B, D.A. Skinfold equations for estimation of body fatness in children and youths. Hum Biol. 1988;60:709-23.

18- Chan YH. Biostatistics 201: Linear regression anaysis. Singapore Med J. 2004;45(2):55-61.

19- Cohen J. Statistical Power Analysis for the behavioral sciences. Second. Associates LE, editor. New York; 1988. $227 \mathrm{p}$.

20- Abbad G, Torres CV. Regressão múltipla stepwise e hierárquica em Psicologia Organizacional: aplicações, problemas e soluções. Estud Psicol. 2002;7:19-29.

21- Faigenbaum AD, Lloyd RS, MacDonald J, Myer GD. Citius, Altius, Fortius beneficial effects of resistance training for young athletes: Narrative review. $\mathrm{Br} J$ Sports Med [Internet]. 2016;50(1):3-7. Available from $\begin{array}{llll}\mathrm{Br} & \mathrm{J} & \text { Sports Med [Internet]. 2016;50(1):3-7. Availah } \\ \text { http://bjsm.bmj.com/lookup/doi/10.1136/bjsports-2015-094621 }\end{array}$

22- Taanila H, Hemminki AJ, Suni JH, Pihlajamäki H, Parkkari J. Low physical fitness is a strong predictor of health problems among young men: a followup study of 1411 male conscripts. BMC Public Health [Internet] 2011;11(1):590. Available from: http://www.biomedcentral.com/1471 $2458 / 11 / 590$

23- Tamura A Akasaka $\mathrm{K}$, Otsudo $T$ Sawada $Y$ Okubo $Y$ Shiozawa J, et al. Fatigue Alters Landing Shock Attenuation During a Single-Leg Vertical Drop Jump. Orthop J Sport Med [Internet]. 2016;4(1):1-7. Available from: http://ojs.sagepub.com/lookup/doi/10.1177/2325967115626412

24- Weeks BK, Carty CP, Horan S a. Effect of sex and fatigue on single leg squat kinematics in healthy young adults. BMC Musculoskelet Disord [Internet]. 2015;16(1):271. Available from: http://www.pubmedcentral.nih.gov/articlerender.fcgi?artid=4590784\&tool=p mcentrez\&rendertype=abstract

25- Benjaminse A, Habu A, Sell TC, Abt JP, Fu FH, Myers JB, et al. Fatigue alters lower extremity kinematics during a single-leg stop-jump task. Knee Surgery, Sport Traumatol Arthrosc. 2008;16(4):400-7.

26- Carden PPJ, Izard RM, Greeves JP, Lake JP, Myers SD. Force and acceleration characteristics of military foot drill: implications for injury risk in recruits. BMJ Open Sport Exerc Med [Internet]. 2015;1(1):bmjsem-2015$000025 . \quad$ Available from: http://bmjopensem.bmj.com/lookup/doi/10.1136/bmjsem-2015-000025

27- Artero EG, España-Romero V, Castro-Piñero J, Ruiz JR, Jiménez-Pavón $D$, Aparicio VA, et al. Criterion-related validity of field-based muscula fitness tests in youth. J Sports Med Phys Fitness. 2012;52(3):263-72.

28- Baur DM, Christophi CA, Tsismenakis AJ, Cook EF, Kales SN Cardiorespiratory Fitness Predicts Cardiovascular Risk Profiles in Career Firefighters. J Occup Environ Med [Internet]. 2011;53(10):1155-60. Available Available
http://content. wkhealth.com/linkback/openurl?sid=WKPTLP:landingpage\&a $\mathrm{n}=00043764-201110000-00010$

29- Kovac M, Jurak G, Strel J, Bednarik J. Comparison of motor development of boys and girls aged 11-17. J Hum Kinet. 2003;10:63-76. 